Journal for ImmunoTherapy of Cancer

\title{
Temozolomide antagonizes oncolytic immunovirotherapy in glioblastoma
}

\author{
Dipongkor Saha (1) ,' Samuel D Rabkin,, ${ }^{2,3}$ Robert L Martuza ${ }^{2,3}$
}

To cite: Saha D, Rabkin SD, Martuza RL. Temozolomide antagonizes oncolytic immunovirotherapy in glioblastoma. Journal for ImmunoTherapy of Cancer 2020;8:e000345. doi:10.1136/ jitc-2019-000345

Accepted 04 May 2020

Check for updates

(c) Author(s) (or their employer(s)) 2020. Re-use permitted under CC BY-NC. No commercial re-use. See rights and permissions. Published by BMJ.

1 Immunotherapeutics and Biotechnology, Texas Tech University Health Sciences Center - Abilene Campus, Abilene, Texas, USA

${ }^{2}$ Neurosurgery, Massachusetts General Hospital, Boston, Massachusetts, USA

${ }^{3}$ Harvard Medical School, Boston, Massachusetts, USA

Correspondence to Dr Dipongkor Saha; Dipongkor.Saha@ttuhsc.edu

\begin{abstract}
Background Temozolomide (TMZ) chemotherapy is a current standard of care for glioblastoma (GBM), however it has only extended overall survival by a few months. Because it also modulates the immune system, both beneficially and negatively, understanding how TMZ interacts with immunotherapeutics is important. Oncolytic herpes simplex virus (oHSV) is a new class of cancer therapeutic with both cytotoxic and immunostimulatory activities. Here, we examine the combination of TMZ and an oHSV encoding murine interleukin $12, \mathrm{G} 47 \Delta$-mIL12, in a mouse immunocompetent GBM model generated from non-immunogenic 005 GBM stem-like cells (GSCs.

Methods We first investigated the cytotoxic effects of $\mathrm{TMZ}$ and/or G47 $\Delta$-IL12 treatments in vitro, and then the antitumor effects of combination therapy in vivo in orthotopically implanted 005 GSC-derived brain tumors. To improve TMZ sensitivity, $0^{6}$-methylguanine DNA methyltransferase (MGMT) was inhibited. The effects of TMZ on immune cells were evaluated by flow cytometery and immunohistochemistry.

Results The combination of TMZ+G47 $\Delta$-IL12 kills 005 GSCs in vitro better than single treatments. However, TMZ does not improve the survival of orthotopic tumor-bearing mice treated with $\mathrm{G} 47 \Delta-\mathrm{IL} 12$, but rather can abrogate the beneficial effects of G47 $\Delta-\mathrm{IL} 12$ when the two are given concurrently. TMZ negatively affects intratumor $T$ cells and macrophages and splenocytes. Addition of MGMT inhibitor $0^{6}$-benzylguanine (06-BG), an inactivating pseudosubstrate of MGMT, to TMZ improved survival, but the combination with G47 $\Delta$-IL12 did not overcome the antagonistic effects of TMZ treatment on oHSV therapy. Conclusions These results illustrate that chemotherapy can adversely affect oHSV immunovirotherapy. As TMZ is the standard of care for GBM, the timing of these combined therapies should be taken into consideration when planning oHSV clinical trials with chemotherapy for GBM.
\end{abstract}

\section{INTRODUCTION}

Glioblastoma (GBM) is the most malignant adult primary brain tumor, invariably lethal with a median survival of about 15 months ${ }^{1}$; despite the advances in current standards of care (surgical resection, radiotherapy, and chemotherapy) and other treatment options, such as antiangiogenic agents and immunotherapy. ${ }^{12}$ GBM is a highly immunosuppressed tumor that is relatively resistant to immunotherapy, including immune checkpoint inhibitors. ${ }^{3}{ }^{4}$ Chemotherapeutic temozolomide (TMZ), a DNA alkylating agent and immunomodulator, ${ }^{5}$ is part of the current standard of care. ${ }^{6}$ Resistance to TMZ is typically due to expression of $\mathrm{O}^{6}$ methylguanine DNA methyltransferease (MGMT), a protein that removes methyl adducts, that can be blocked by inactivating pseudosubstrates such as $\mathrm{O}^{6}$-benzylguanine $(\mathrm{O} 6-\mathrm{BG}){ }^{7}$ GBM tumors are very heterogeneous; containing a subpopulation of GBM stem-like cells (GSCs) or tumor-initiating cells that are believed to be responsible for treatment failure, tumor recurrence, and immune escape, making them critical targets for therapy. ${ }^{8-11}$ Patient-derived GSC models recapitulate GBM histology in immunodeficient mice, ${ }^{12}$ but these models are not useful to examine host immune responses. We recently developed an immunocompetent mouse GSC-derived (005) orthotopic brain tumor model, which recapitulates both GBM histology and immunosuppression. ${ }^{13-16}$

Oncolytic viruses (OVs) are a new class of anticancer agent, which selectively kill cancer cells (oncolysis), including cancer stem cells, and induce antitumor immunity. ${ }^{17-20}$ Among OVs, oncolytic herpes simplex virus (oHSV) is the furthest along in the clinic, with talimogene laherparepvec approved for use in advanced melanoma. ${ }^{21}$ G47 4 , an oHSV currently in a registration clinical trial for recurrent GBM in Japan, was not efficacious alone in the 005 GSC model, however, intratumoral viral expression of murine interleukin 12 with G47D-mIL12 improved antitumor immunity, but median survival was only modestly extended. ${ }^{13}$ Thus, additional combination strategies are needed to improve the therapeutic outcome of oncolytic immunovirotherapy. ${ }^{21}$ TMZ synergizes with $\mathrm{OVs}^{22-27}$ and different forms of immunotherapy. ${ }^{28-31}$ We previously reported that TMZ synergized with G47 $\triangle$ in killing human GSCs in vitro and human GSC-derived orthotopic tumors in immunodeficient mice. ${ }^{24}$ Only a few of these combination studies (TMZ+OV) were with immunocompetent GBM models. Because 
TMZ is a standard of care for GBM and has immunomodulatory consequences, it is important to examine how TMZ treatment affects oHSV efficacy in immunocompetent GBM models, which may reflect features of the clinical situation.

\section{MATERIALS AND METHODS \\ Cells, virus, and chemotherapeutic drugs}

Mouse 005 GSCs, a gift from Dr I Verma (Salk Institute, San Diego), were established from GBM generated with lentiviral transduction of $\mathrm{H}$-Ras and activated Akt in

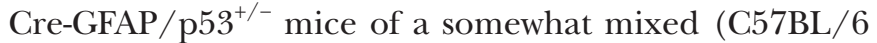
and some $\mathrm{FVB} / \mathrm{N}$ ) background. ${ }^{32}$ They were cultured as spheres in serum-free stem cell medium composed of advanced DMEM/F12 (ThermoFisher Scientific), supplemented with $2 \mathrm{mM} \mathrm{L-glutamine} \mathrm{(Corning),} 1 \%$ N2 supplement (ThermoFisher Scientific), $2 \mu \mathrm{g} / \mathrm{mL}$ heparin (Sigma-Aldrich), 0.5\% penicillin G-streptomycin sulfateamphotericin B complex (Corning), recombinant human EGF (20 ng/mL; R\&D Systems), and recombinant human FGF-basic (20 ng/mL; Peprotech), as described, ${ }^{13} 14$ and dissociated with Accutase (Innovative Cell Technologies) for passaging. Low-passage cells were used, which were free from mycoplasma (LookOut mycoplasma kit; Sigma).

G47D-mIL12 was constructed from G47D (deletions in $\gamma 34.5$ and $\alpha 47$ genes and inactivating insertion of LacZ into ICP $6^{33}$ ) by insertion of CMV IE promoterdriven mouse IL-12 cDNA, $^{13}$ and grown in Vero cells after low multiplicity of infection (MOI), and purified as described. ${ }^{34}$

O6-BG (Sigma-Aldrich) and TMZ (Sigma-Aldrich) were dissolved in dimethyl sulfoxide (DMSO) for in vitro studies, and diluted in 005 GSC culture medium before adding to cells.

\section{Cytotoxicity assays}

Dissociated mouse 005 GSCs were seeded into 96-well cell culture plates (2000 cells/well). For virus studies, cells were plated using 005 medium with 'no heparin', and TMZ and/or virus, diluted in 005 medium without heparin, added to cells at indicated doses immediately after seeding. O6-BG $(5 \mu \mathrm{M})$ was added to cells 1 hour prior to TMZ treatment. Two hours post-treatment, medium 'with heparin' was added and incubated for 4 days at $37^{\circ} \mathrm{C}$ before MTS assays were performed following manufacturer's instruction (Promega). Each experiment was repeated at least two independent times and performed in triplicate.

\section{Short-hairpin RNA (shRNA)-mediated knockdown of MSH6}

shRNA-mediated knockdown of MSH6 in 005 GSCs was performed as previously described. ${ }^{24}$ Briefly, plasmid construct containing shRNA sequences against MSH6 mRNA (TRCN0000071163, designated shRNA 1; TRCN0000071164, designated shRNA 2; TRCN0000071165, designated shRNA
3; TRCN0000071166, designated shRNA 4; TRCN0000071167, designated shRNA 5) were purchased from Dharmacon or non-targeting shRNA (SHC002) from Sigma. Generation of lentiviral constructs, lentiviral transduction of 005 GSCs for MSH6 knockdown, and selection with puromycin were performed as previously described.$^{24}$ MSH6 knockdown was performed at least two times and the level of target gene was assessed by western blot. MTS cytotoxicity assays in MSH6 knockdown cells were performed as described above.

\section{Animal studies}

C57BL/6 mice (7-8 weeks old) were obtained from the National Cancer Institute (Frederick, Maryland). All mouse procedures were approved by the Institutional Animal Care and Use Committee at the Massachusetts General Hospital. Dissociated 005 GSCs $\left(2 \times 10^{4}\right)$ were implanted stereotaxically into the striatum $(2.2 \mathrm{~mm}$ lateral from Bregma and $2.5 \mathrm{~mm}$ deep) on day 0 to generate orthotopic intracranial tumors. Mice were randomly divided into groups at day 7 and treated with G47 $\Delta$-mIL12 $\left(5 \times 10^{5} \mathrm{pfu}\right.$ in $2 \mu \mathrm{L} /$ mouse $)$ or phosphate buffered saline (PBS) injected intratumorally at the same stereotaxic coordinates on day 12 , and TMZ $(7.5 \mathrm{mg} / \mathrm{kg}$ dissolved in $0.93 \%$ DMSO or 25 or $50 \mathrm{mg} / \mathrm{kg}$ dissolved in $3.1 \%$ DMSO) or vehicle solution injected intraperitoneally from days 10 to 14 . For O6-BG+TMZ+Virus combination studies, O6-BG $(0.3 \mathrm{mg} /$ mouse dissolved in $40 \%$ polyethylene glycol-400 (Sigma) in PBS) or vehicle solution was injected intraperitoneally 1 hour prior to TMZ administration (as in Kanai $e t a l^{24}$ ). Mice were followed for neurological symptoms and euthanized before becoming moribund. Animal caretakers were blinded to the treatment knowledge.

\section{Immunohistochemistry for tumor-infiltrating immune cells}

C57BL/ 6 mice implanted with 005 GSCs were treated with TMZ from days 19 to 23. On day 26 mice were sacrificed, brains removed and fixed in $10 \%$ formalin, embedded in paraffin, and $5 \mu \mathrm{m}$ sections subjected to immunohistochemical staining with primary antibodies against CD4 (anti-mouse CD4; eBioscience, Cat. \# 14-9766-80), CD8 (anti-mouse CD8a; eBioscience, Cat. \# 14-0808-80), or CD68 (anti-CD68; Abcam, Cat. \# ab125212), followed by incubation with appropriate secondary antibodies (HRP anti-rat or anti-rabbit IgG; Vector Laboratories), as described previously. ${ }^{1635}$ The number of positive cells were counted from three random fields/tumor section (one section/mouse). Counter was blinded to the treatments.

\section{Multicolor flow cytometry}

For 10-color flow cytometric analysis, single cell suspensions from harvested spleens were prepared (as in Cheema et $a l^{13}$ ) and stained with fluorochrome-conjugated antimouse antibodies (PerCP-Cy-5.5 anti-mouse CD4, PE-Cy7 anti-mouse CD69, Alexa Fluor 647 anti-mouse FoxP3, Brilliant Violet 510 anti-mouse CD8a, Brilliant Violet 421 anti-mouse NK 1.1, Brilliant Violet 605 anti-mouse/ 

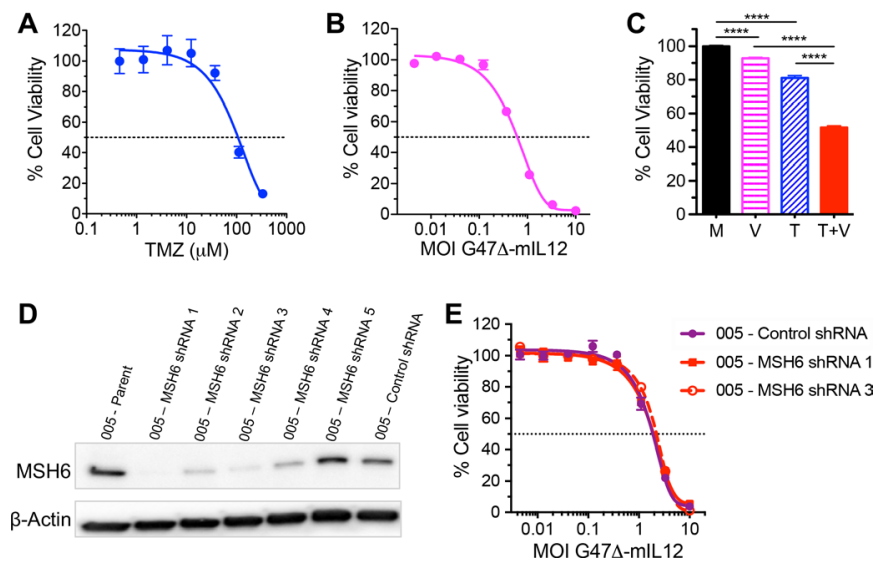

Figure 1 Cytotoxic effects of TMZ and/or G47D-IL12 in vitro. Dose-response curves for TMZ (A) and G47 $\triangle$-IL12 (B) 4 days after treatment, as measured by MTS assay. (C) 005 GSCs were treated with $\sim 1 \mathrm{C}_{20}$ dose of $\mathrm{TMZ}(40 \mu \mathrm{M})$ and/ or $\sim \mathrm{IC}_{10}$ dose of G47 $\Delta$-mIL12 (MOI 0.1) for 4 days, and cell viability assessed by MTS assay. Each graph represents an average of three experiments performed in triplicate. Data presented as mean \pm SEM. ${ }^{\star \star \star *} \mathrm{p}<0.0001$ between indicated groups (unpaired two-tailed Student's t-test). M, mock; V, G47 $\Delta$-mIL12 virus; T, TMZ; and T+V, TMZ+G47 $\Delta$-mIL12. (D, E) Effects of MSH6 knockdown on 005 GSCs sensitivity to G47D-IL12. (D) 005 GSCs were lentivirally transduced with MSH6 (five independent sequences) or non-targeting (control) shRNAs. MSH6 protein levels were detected by western blot. Beta-actin was used as a loading control. (E) Dose-response curves for G47D-IL12 in MSH6 (005-MSH6 shRNA 1 and 005-MSH6 shRNA 3) or control (005-control shRNA cells) knockdown cells at 4 days post virus treatment, as measured by MTS assay. GBM, glioblastoma; GSCs, GBM stem-like cells; MOI, multiplicity of infection; shRNA, short-hairpin RNA; $\mathrm{TMZ}$, temozolomide.

human CD11b, APC-Cy7 anti-mouse CD11c, Alexa Fluor 700 anti-mouse Ly-6G/Ly-6C Gr-1, and FITC anti-mouse CD19), as well as appropriate isotype control antibodies, as described. ${ }^{14}$ All antibodies were obtained from Biolegend. Zombie UV live/dead fixable viability kit was used to stain dead cells. We followed a 'no-wash' sequential staining protocol (Biolegend) to stain dead cells and for surface staining. Intracellular FoxP3 staining was performed following the FoxP3 intracellular staining protocol (Biolegend). Fluorescent minus one and singlecolor compensation controls were included for each color, as we described. ${ }^{1436}$ All samples were run in a LSRII flow cytometer (BD Biosciences) and flow cytometric data were analyzed by FlowJo software V.10.5.3 (Tree Star). Technician acquiring and gating the data were blinded to the treatments.

\section{Western blot}

005 GSCs were pelleted and lysed in radioimmunoprecipitation buffer (Boston Bioproducts) with a cocktail of protease and phosphatase inhibitors (Roche). Protein $(20 \mu \mathrm{g})$ was separated by $4 \%-15 \%$ sodium dodecyl sulfate-polyacrylamide gel electrophoresis (SDS-PAGE), and transferred to polyvinylidene difluoride (PVDF) membranes by electroblotting. Western blotting was performed with primary antibodies to MGMT (Biovision, Cat. \# 3820-100) and MSH6 (Cell Signaling Technology, Cat. \# 3995). Primary antibodies to $\beta$-actin (Cell Signaling Technology, Cat. \# 4970) and Vinculin (Cell Signaling Technology, Cat. \# 13901) were used as loading controls.

\section{Statistical analysis}

Dose-response curves and $\mathrm{IC}_{50}$ values were calculated using Prism 7 GraphPad software V.7.0e. Cell viabilities at each time point, positive cell counts for each group (immunohistochemistry), and flow cytometry were compared by unpaired two-tailed Student's t-test. One-way analysis of variance followed by Tukey's multiple comparison test was used to compare bodyweights between indicated treatments. Survival data were analyzed by Kaplan-Meier survival curves, and comparisons were performed by logrank test. A p value of $<0.05$ was considered significant. All statistical analyses were performed using Prism 7 GraphPad software V.7.0e.

\section{RESULTS}

\section{In vitro sensitivity of 005 GSCs}

We first investigated the cytotoxic effects of TMZ and/or G47D-IL12 treatments in vitro. 005 GSCs are somewhat sensitive to TMZ $\left(\mathrm{IC}_{50} \sim 100 \mu \mathrm{M}\right.$; figure $\left.1 \mathrm{~A}\right)$ and $\mathrm{G} 47 \Delta$ IL12 $\left(\mathrm{IC}_{50}=\right.$ MOI 0.7; figure $\left.1 \mathrm{~B}\right)$, as determined by MTS assay. In patients, TMZ treatment results in peak tumor concentrations of $<40 \mu \mathrm{M} .^{37}$ To evaluate combination effects, 005 GSCs were treated with $\sim \mathrm{IC}_{20}$ of TMZ $(40 \mu \mathrm{M})$, $\sim \mathrm{IC}_{10}$ of G47A-IL12 (MOI 0.1 ), or both. Viability of 005 GSCs was significantly reduced after each single treatment compared with mock $(\mathrm{p}<0.0001)$, and the combination (TMZ+G47 $\Delta$-IL12) was significantly better than either agent alone $(p<0.0001$, figure $1 C)$. In mouse 005 GSCs, knockdown of the MSH6 gene, which is associated with TMZ resistance in $\mathrm{GBM}^{24}$ did not alter their sensitivity to oHSV as compared with control shRNA treated 005 cells (figure 1D-E).

\section{Combination therapy in vivo}

Because the combination significantly improved GSC killing in vitro, we hypothesized that the combination would induce superior antitumor efficacy in vivo, as seen with human GSCs in immunodeficient mice. ${ }^{24} \mathrm{TMZ}$ dosing regimens can vary from 75 to $200 \mathrm{mg} / \mathrm{m}^{2} /$ day in patients with GBM. ${ }^{37}$ According to the Medscape body surface area-based dosing conversion, $75 \mathrm{mg} / \mathrm{m}^{2} /$ day is equal to $\sim 125 \mathrm{mg}$ /day for $60 \mathrm{~kg}$ bodyweight, which is equivalent to $25 \mathrm{mg} / \mathrm{kg}$ for a $20 \mathrm{~g}$ mouse, according to species to species dosing conversion described in Freireich et al. ${ }^{38}$ To test the combination in vivo, mice bearing orthotopic allografts of 005 GSC-derived tumors were treated with intraperitoneal TMZ (25 mg/ kg once daily from days 10 to 14 postimplantation) and/or intratumoral G47 $\Delta$-IL12 $\left(5 \times 10^{5} \mathrm{pfu}\right.$ at day 12 , figure $2 \mathrm{~A}$, lower), and animals followed until they became moribund. No extension of survival was seen with 

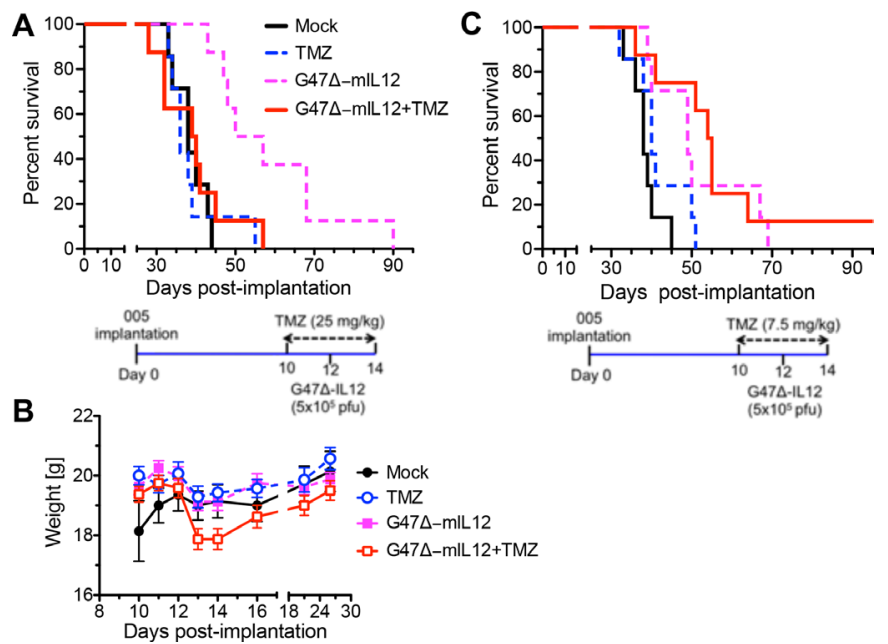

Figure $2 \mathrm{TMZ}$ treatment in combination with intratumoral G47D-IL12 in C57BL/6 mice bearing orthotopic mouse 005 GSC-derived brain tumors. (A) Kaplan-Meier survival curve. C57BL/6 mice, implanted with mouse 005 GSCs on day 0, were treated with $\mathrm{TMZ}(25 \mathrm{mg} / \mathrm{kg})$ or vehicle intraperitoneally from days 10 to 14 , and G47 $\triangle$-mIL12 $\left(5 \times 10^{5}\right.$ pfu) or PBS injected intratumorally on day 12 ( $n=7$ for mock and TMZ, and $n=8$ for $\mathrm{G} 47 \Delta$-IL12 and combination). Schema illustrated below. (B) Bodyweight of tumor-bearing mice (from A) after treatment. Mean \pm SEM, no significant differences between treated and mock (one-way ANOVA, Tukey's test post-test). (C) Kaplan-Meier survival curve for low-dose TMZ (7.5 mg/ $\mathrm{kg}$ ); same experimental schedule as in (A). $n=7$ for mock and single treatments, $n=8$ for combination. Schema illustrated below. The long-term surviving mouse from the combination group was sacrificed on day 223 , and tumor was not present. The median survival of mock (38 days) is significantly different from G47 $\Delta$-IL12 (median survival=49 days, $p=0.003$ ) or combination (median survival $=54.5$ days, $\mathrm{p}=0.002$ ); $\mathrm{TMZ}$ (median survival=40 days) is significantly different from combination $(p=0.0085)$. No other significant differences were observed. ANOVA, analysis of variance; GBM, glioblastoma; GSCs, GBM stem-like cells; TMZ, temozolomide.

TMZ treatment alone (median survival $=36$ days; $\mathrm{p}=0.81$ ), while G47 $\Delta$-IL12 treatment alone significantly extended survival (median survival=53.5 days) compared with mock (median survival=38 days; $\mathrm{p}=0.0002$ ). Surprisingly, combination therapy abrogated the efficacy of G47 $\Delta$-IL12, with the median survival of the combination group (39.5 days) similar to the mock group (38 days, figure 2A). We followed bodyweight to evaluate treatment toxicity. While there was a dip in median weight at days 13 and 14, after virus treatment, it was not significantly different from Mock (figure 2B).

Low-dose TMZ $(7.5 \mathrm{mg} / \mathrm{kg})$ treatment has been shown to enhance oncolytic adenovirus therapy in a relatively immunogenic GL261 orthotopic brain tumor model. ${ }^{25}$ Therefore, we tested whether low-dose TMZ would improve the therapeutic outcome of G47 $\Delta$-IL12 therapy, using the same schema (figure 2C, lower) as in figure 2A. Low-dose TMZ did not improve survival alone or when used in combination with G47 $\Delta$-IL12 (figure 2C), but did not abrogate the therapeutic efficacy of G47 $\Delta$-IL12, as with $25 \mathrm{mg} / \mathrm{kg}$ (figure $2 \mathrm{~A}$ ).

\section{TMZ effects on immune cells}

To try to understand how TMZ is altering the immune response, we examined the effects of TMZ locally on brain tumor-infiltrating immune cells and systemically in the spleen. Mice bearing 005 GSC-derived tumors were treated with TMZ ( $7.5 \mathrm{mg}$ or $25 \mathrm{mg} / \mathrm{kg}$ ) from days 19 to 23 postimplantation, and brains and spleens collected 3 days later (day 26). Formalin-fixed paraffin-embedded brain tumor sections were subjected to immunohistochemistry for tumor-infiltrating immune cells, and spleens mechanically dissociated to single cells for multicolor flow cytometry (as in Cheema et $a l^{13}$ ). In the tumor, lowdose TMZ significantly reduced the number of $\mathrm{CD}^{+}$and $\mathrm{CD}^{+}$tumor infiltrating lymphocytes (TILs) by 1.7 -fold and 2.7-fold, respectively, and $\mathrm{CD} 68^{+}$tumor-associated

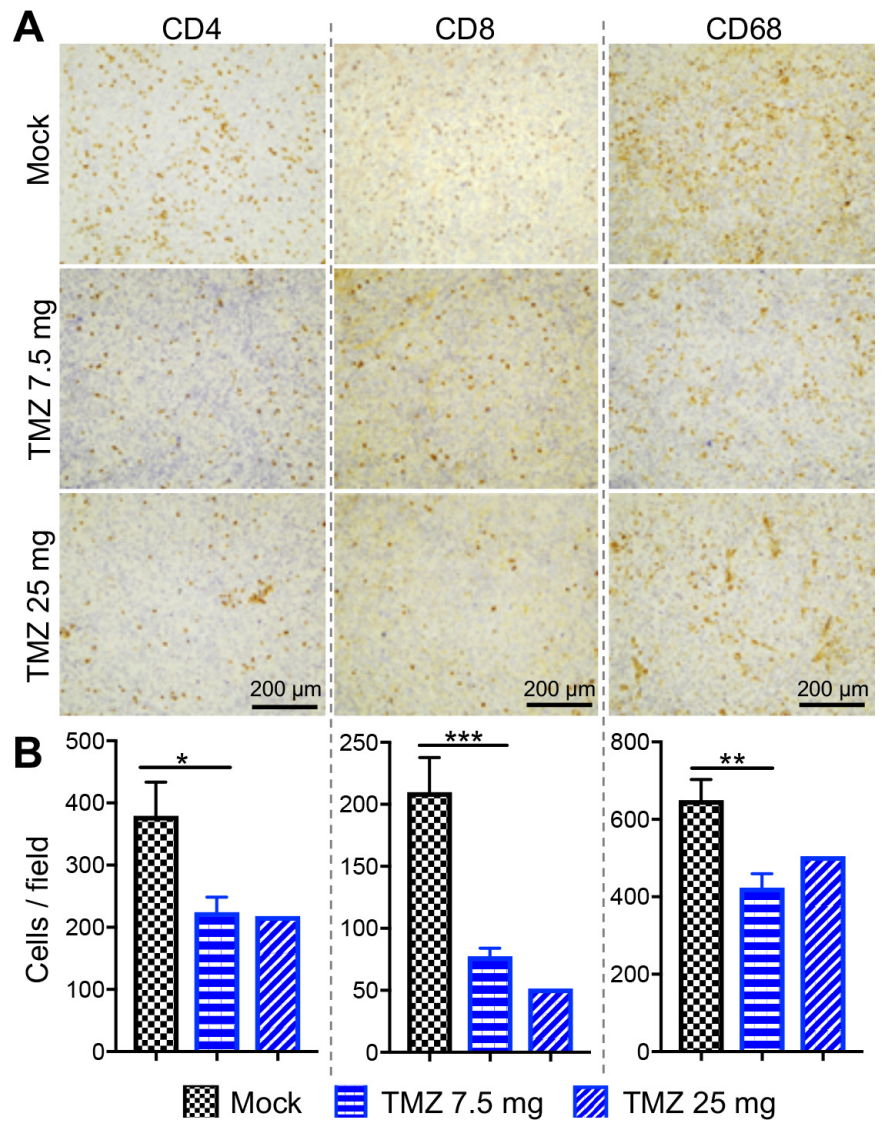

Figure 3 Immunohistochemical analysis of tumor-infiltrating immune cells. C57BL/6 mice implanted with 005 GSCs on day 0 , and treated with $\mathrm{TMZ}$ ( 7.5 or $25 \mathrm{mg} / \mathrm{kg}$ ) or vehicle solution intraperitoneally from days 19 to 23 . Three days after the last TMZ treatment (day 26), mice were sacrificed and sections subjected to immunohistochemical staining for CD4, CD8, and CD68. $\mathrm{n}=4$ for mock, $\mathrm{n}=3$ for $\mathrm{TMZ}, 7.5 \mathrm{mg} / \mathrm{kg}$ group, and $\mathrm{n}=2$ for $\mathrm{TMZ} 25 \mathrm{mg} / \mathrm{kg}$ group. (A) Representative images with positive cells stained brown; scale bar $=200 \mu \mathrm{m}$. (B) The number of positive cells were counted. Mean \pm SEM of all fields. ${ }^{*} p<0.05,{ }^{* *} p<0.01,{ }^{* \star *} p<0.001$ (unpaired two-tailed Student's t-test). GBM, glioblastoma; GSCs, GBM stem-like cells; TMZ, temozolomide. 


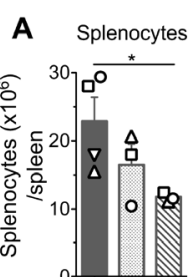

$\mathbf{F}$

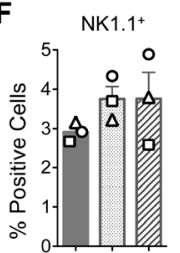

B $\mathrm{CD4}^{+}$

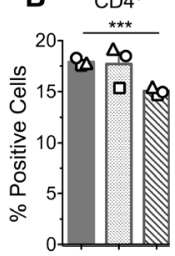

G $\mathrm{CD}_{11 \mathrm{~b}^{+}}$

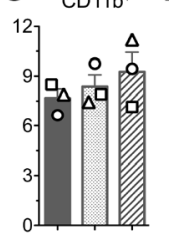

Mock

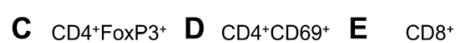

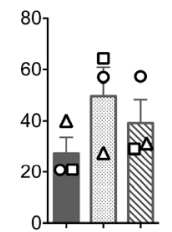

$\mathbf{H}_{\mathrm{CD} 11 \mathrm{~b}^{+} \mathrm{CD} 11 \mathrm{c}^{+}}$

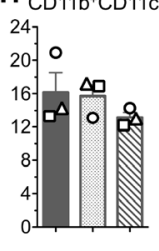

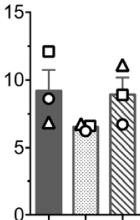

I $\mathrm{CD}_{11 \mathrm{~b}^{+} \mathrm{Gr} 1^{+}}$

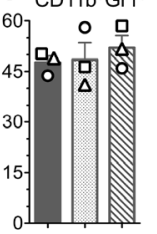

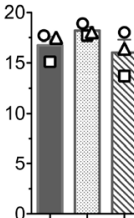

J $\mathrm{CD} 19^{+}$

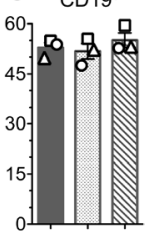

Figure 4 FACS analysis of splenocytes following TMZ treatment. The same experimental mice as in figure 3. On day 26 , when mice were sacrificed, spleens were harvested, total number of splenocytes/spleen counted and subjected to 10-color flow cytometry staining. (A) Average number of splenocytes (mean \pm SEM) from all mice $(n=3$ for $T M Z$ group and $n=4$ for mock). (B-J) Splenocytes stained with fluorochrome-conjugated anti-mouse antibodies/dye and multicolor fluorescence-activated cell sorting (FACS) performed. Bar graphs of the percentages of live sorted positive cells presented with symbols indicating individual mice. Mean $\pm S E M{ }^{*} p<0.05,{ }^{* \star *} p<0.001$ (unpaired two-tailed Student's t-test). TMZ, temozolomide

macrophages (TAMs) by 1.5-fold compared with the mock group (figure 3A-B). Higher dose TMZ, while not significant, also reduced CD4 ${ }^{+}$TILs (1.7-fold) and TAMs (1.3-fold), and CD8 ${ }^{+}$TILs (4-fold) compared with mock (figure 3A-B). In the spleen, only higher dose TMZ significantly reduced total splenocytes (figure $4 \mathrm{~A}$ ) and $\mathrm{CD} 4^{+} \mathrm{T}$ cells (figure 4B). Other immune cells, such as regulatory $\left(\mathrm{CD} 4^{+} \mathrm{FoxP}^{+}\right)$and activated $\mathrm{T}$ cells $\left(\mathrm{CD} 4^{+} \mathrm{CD} 69^{+}\right)$, $\mathrm{CD}^{+} \mathrm{T}$ cells, natural killer cells $\left(\mathrm{NK} 1.1^{+}\right)$, myeloid cells $\left(\mathrm{CD} 11 \mathrm{~b}^{+}\right)$, dendritic cells $\left(\mathrm{CD} 11 \mathrm{~b}^{+} \mathrm{CD} 11 \mathrm{c}^{+}\right)$, monocytes $\left(\mathrm{CD} 11 \mathrm{~b}^{+} \mathrm{Gr} 1^{+}\right)$, and B cells $\left(\mathrm{CD} 19^{+}\right)$were not significantly altered by either TMZ dose (figure 4C-J).

\section{Inhibiting MGMT}

005 GSCs express MGMT (figure 5A), which is associated with TMZ resistance. ${ }^{39} 40$ If MGMT expression in 005 GSCs contributes to TMZ resistance, its inhibition should improve efficacy. ${ }^{24}$ O6-BG, an inactivating pseudosubstrate of MGMT, significantly reduced MGMT expression (figure 5A), as seen previously in oral cancer cells, ${ }^{41}$ and sensitized 005 GSCs to TMZ, shifting the $\mathrm{IC}_{50}$ by $\sim 5$-fold (figure 5B). O6-BG also sensitized 005 GSCs to the combination treatment (TMZ+G47 $\Delta$-IL12) by $\sim 2$ fold compared with no O6-BG (figure 5C). Because O6-BG significantly reduced the $\mathrm{IC}_{50}$ dose of $\mathrm{TMZ}$ in vitro (figure $5 \mathrm{~B}$ ) to a dose within the peak tumor concentrations in patients, ${ }^{37}$ we hypothesized that O6-BG treatment would improve the efficacy of TMZ treatment alone or in combination with G47A-IL12 in vivo. The rationale for the combination with MGMT inhibitor was that TMZ did not inhibit 005 tumor growth alone, and therefore it was possible that TMZinduced cell death was necessary for immune-mediated effects that might enhance TMZ+G47 $\Delta$-IL12 combination effects. Mice bearing 005 GSC-derived tumors were treated with TMZ (50 mg/kg) and O6-BG or vehicle, and/ or G47A-IL12 as illustrated in the schema (figure $5 \mathrm{D}$ ). The increased sensitivity of 005 GSCs in vitro to TMZ following O6-BG treatment was reproduced in vivo, with the median survival of the O6-BG+TMZ treatment group marginally, but significantly longer than TMZ alone (40 days vs 35 days; $p=0.02$, figure $5 \mathrm{D}$ ). As in figure $2 \mathrm{~A}$, TMZ combined with G47 $\Delta$-IL12 (median survival=42 days) significantly $(p=0.0004)$ abrogated the beneficial effects of oncolytic G47 $\Delta$-IL12 treatment (median survival=70 days). Unfortunately, even with the chemotherapeutic effects on tumor growth, the addition of O6-BG to the combination of TMZ+G47A-IL12 (median survival=42 days) did not overcome the antagonistic effects of TMZ treatment on oHSV therapy (figure 5D). There was no significant difference in bodyweights between any of the treatment groups and mock (figure 5E).

\section{DISCUSSION}

There is conflicting and limited data on the immunological consequences of standard of care TMZ dosing schedules on immunotherapy. ${ }^{5} 42$ Here, we examined the effects of different TMZ doses on oHSV immunovirotherapy in a representative mouse immunocompetent GBM model. Most of the preclinical studies of GBM immunotherapy have been performed with the GL261 mouse glioma model. Unfortunately, G1261 is relatively immunogenic ${ }^{434}$ and minimally sensitive to TMZ in vitro, ${ }^{28} 45$ in contrast to the 005 model. ${ }^{13} 14$

We did not expect low-dose TMZ $(7.5 \mathrm{mg} / \mathrm{kg})$ to inhibit tumor growth because it would lead to serum concentrations well below the sensitivity of 005 GSCs. However, it did have a significant impact on TILs and TAMs (figure 3). We previously showed that curative therapy with G47 $\Delta$-mIL12 in combination with anti-PD-1 and anti-CTLA- 4 was dependent on both $\mathrm{CD} 4^{+}$and $\mathrm{CD}^{+} \mathrm{T}$ cells and macrophages. ${ }^{14} 46$ In the spleen, $\mathrm{CD} 4^{+} \mathrm{T}$ cells were significantly reduced after $25 \mathrm{mg} / \mathrm{kg} \mathrm{TMZ}$, with a trend, not significant, to increased Tregs (figure 4C). In the GL261 model, low-dose TMZ significantly increased peripheral blood NK cells and their activation and tumor infiltration. ${ }^{47}$ We found a trend, not significant, towards increased NK cells in the spleens of TMZ-treated mice (figure 4F). Increased NK cells might improve antitumor activity, ${ }^{48}$ but may also inhibit oHSV efficacy. ${ }^{49}$ The effect of TMZ on immunotherapy seems to be somewhat dose and schedule dependent, which we did not evaluate. In the GL261 glioma model, the survival advantage of antiPD-1 therapy was abrogated with 'standard-dosing' of TMZ (50 mg/kg), but preserved with metronomic $25 \mathrm{mg} /$ $\mathrm{kg}$ TMZ. ${ }^{50}$ Here, both 25 and $50 \mathrm{mg} / \mathrm{kg}$ TMZ abrogated G47 $\Delta$-mIL12 antitumor efficacy. The lack of a large difference between immune cell phenotypes at $7.5 \mathrm{mg} / \mathrm{kg}$, with 

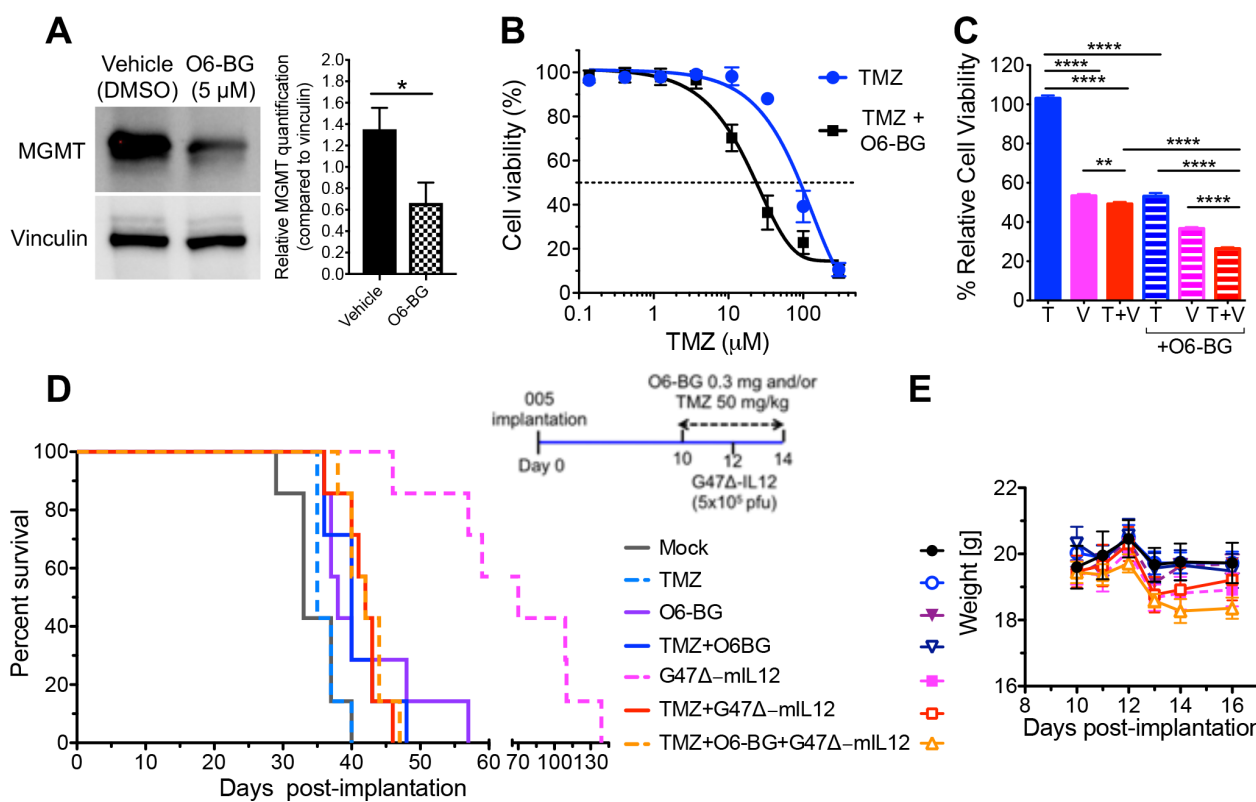

$\mathbf{E}$

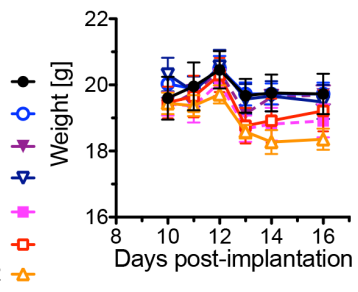

Figure 5 Inhibition of MGMT with O6-BG does not overcome TMZ antagonism to G47A-mIL12 treatment in vivo. (A) Western blot and densitometry of 005 GSC lysates for MGMT. Vinculin was used as loading control. 005 GSCs were treated with or without O6-BG $(5 \mu \mathrm{M})$ for 1 hour, cell lysates collected and blotted in triplicates. Representative blots are shown. Bar graph represents relative MGMT quantification compared with loading control. Mean $\pm S E M{ }^{*} p<0.05$ (unpaired Student's t-test). (B) Dose-response curves for 005 GSCs after TMZ treatment with or without O6-BG $(5 \mu \mathrm{M})$. The graph represents an average of three experiments performed in triplicate. Mean \pm SEM. (C) 005 GSCs treated with TMZ (T; $10 \mu M)$ and/or G47A-IL12 (V; MOI 0.1), and/or with O6-BG $(5 \mu \mathrm{M})$. The graph represents an average of three experiments and 8 wells/condition. Mean $\pm S E M$. ${ }^{* *} \mathrm{p}<0.01$,

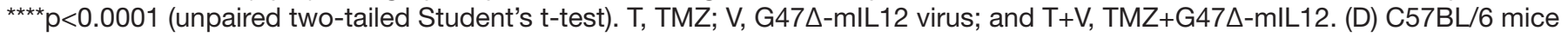
implanted with 005 GSCs were treated with TMZ or vehicle solution intraperitoneally from days 10 to 14 , and G47 -mIL12 or PBS injected intratumorally on day 12 , and O6-BG $(0.3 \mathrm{mg} / \mathrm{mouse})$ or vehicle (right; treatment schema for experiment). $\mathrm{n}=7$ for each treatment group. (left) Kaplan-Meier survival curve with the indicated treatment groups. (E) Bodyweight of tumor-bearing mice after treatment. Mean \pm SEM, no significant differences between treated and mock (one-way ANOVA, Tukey's posthoc test). ANOVA, analysis of variance; GBM, glioblastoma; GSCs, GBM stem-like cells; MOI, multiplicity of infection; TMZ, temozolomide.

no effect on G47 4 -mIL12 efficacy, and $25 \mathrm{mg} / \mathrm{kg}$ suggests that other immune cell types may be important for TMZ immunosuppression of G47A-IL12 activity.

In contrast to human GSCs, where TMZ synergized with oHSV in extending survival of immunodeficient mice with orthotopic tumors, ${ }^{24} \mathrm{TMZ}$ abrogated oHSV efficacy in mouse GSC-derived tumors. HSV has a complicated interaction with the host immune system. ${ }^{17}$ It should be pointed out that G47A replicates well in human GSCs, ${ }^{24}$ but poorly in 005 GSCs. ${ }^{13}$ In human GSCs, oHSV impairs DNA damage responses, promoting synergy with oHSV. ${ }^{2451}$ It is unknown whether this also occurs in mouse GSCs, but it will be important to understand the balance between oncolytic/cytotoxic and immune responses in combination therapy with TMZ. 005 GSCs express MGMT, and MGMT promoter methylation is associated with TMZ resistance. ${ }^{24}$ While the addition of MGMT inhibitor $(\mathrm{O} 6-\mathrm{BG})$ to $\mathrm{TMZ}$ decreased the $\mathrm{IC}_{50}$ of $\mathrm{TMZ}$ in vitro and extended survival, it did not improve the combination with virus. TMZ has been examined in combination with other OVs in immunocompetent glioma models and found to extend survival. ${ }^{25} 2752$ Oncolytic myxoma virus synergized with TMZ $(100 \mathrm{mg} / \mathrm{kg})$ in treating mBTIC0309 GSC-derived tumors in immunocompetent mice, but had no effect in immunodeficient mice, ${ }^{27}$ indicating $\mathrm{T}$ cell-mediated efficacy. The sequence of oncolytic adenovirus and TMZ treatments was shown to play a role in tumor infiltration of T cells and treatment efficacy. ${ }^{25} \mathrm{TMZ}$ $(7.5 \mathrm{mg} / \mathrm{kg})$ significantly extended survival of oncolytic adenovirus-treated GL261 tumors when oncolytic adenovirus was injected prior to TMZ. TMZ treatment was associated with a significant increase in $\mathrm{CD} 4^{+}$TILs on day 8, whereas, we saw a significant decrease at day $3 .{ }^{25}$ We do not know what contributed to the difference in efficacy between the two studies; however, there are a number of possible contributors: the tumor model, somewhat immunogenic GL261 versus non-immunogenic 005; oncolytic adenovirus versus oHSV; the treatment schema, pretreatment with oncolytic adenovirus versus oHSV-IL12 in the middle of TMZ treatment; and/or expression of IL12. It remains to be determined whether any of these differences could improve oHSV+TMZ combination therapy.

\section{CONCLUSIONS}

These results illustrate that chemotherapy can adversely affect oHSV immunovirotherapy. The effects, either antagonistic or neutral, occur over a range of TMZ doses $(7.5-50 \mathrm{mg} / \mathrm{kg})$ and with MGMT inhibitor O6-BG. Interestingly, TMZ had a much larger negative effect on the 
number of intratumoral $\mathrm{T}$ cells and macrophages than splenocyte subsets, indicating a large shift in the tumor microenvironment, especially at low doses. As TMZ is the standard of care for GBM, the scheduling of oHSV injections relative to TMZ treatment in the clinic should be carefully considered.

Acknowledgements We thank Dr. I. Verma and Y. Soda (Salk Institute, San Diego, CA) for providing 005 GSCs. We thank M. Humphrey for assistance with surgeries, HSCI CRM Flow Cytometry Core for their technical assistance with the flow cytometry, and the animal facility personnel for taking care of mice. We also thank the BioLegend Tech Group for their help in choosing the fluorochrome panel.

Contributors DS: performed experiments; DS, RLM, SDR: designed experiments and wrote the manuscript; RLM, SDR: obtained funding.

Funding DS is supported by startup funds from Dodge Jones Foundation-Abilene and TTUHSC-School of Pharmacy. This study is funded in part by grants from NIH (R01NS032677 to RLM and R01CA16072 to SDR) and the Thomas A. Pappas Chair in Neurosciences (SDR).

Competing interests SDR and RLM are inventors on patents relating to oHSV owned and managed by Georgetown University and Massachusetts General Hospital that have been licensed to Amgen, for which they receive royalties.

Patient consent for publication Not required.

Ethics approval All mouse procedures were approved by the Institutional Animal Care and Use Committee at the Massachusetts General Hospital.

Provenance and peer review Not commissioned; externally peer reviewed. Data availability statement Data are available upon reasonable request.

Open access This is an open access article distributed in accordance with the Creative Commons Attribution Non Commercial (CC BY-NC 4.0) license, which permits others to distribute, remix, adapt, build upon this work non-commercially, and license their derivative works on different terms, provided the original work is properly cited, appropriate credit is given, any changes made indicated, and the use is non-commercial. See http://creativecommons.org/licenses/by-nc/4.0/.

\section{ORCID iD}

Dipongkor Saha http://orcid.org/0000-0002-9923-5533

\section{REFERENCES}

1 Delgado-López PD, Corrales-García EM. Survival in glioblastoma: a review on the impact of treatment modalities. Clin Trans/ Oncol 2016;18:1062-71.

2 Alexander BM, Cloughesy TF, Glioblastoma A. Adult glioblastoma.. J Clin Oncol 2017;35:2402-9.

3 Perng P, Lim M. Immunosuppressive mechanisms of malignant gliomas: parallels at non-CNS sites. Front Oncol 2015;5:153.

4 Preusser M, Lim M, Hafler DA, et al. Prospects of immune checkpoint modulators in the treatment of glioblastoma. Nat Rev Neurol 2015;11:504-14.

5 Karachi A, Dastmalchi F, Mitchell DA, et al. Temozolomide for immunomodulation in the treatment of glioblastoma. Neuro Oncol 2018;20:1566-72.

6 Stupp R, Mason WP, van den Bent MJ, et al. Radiotherapy plus concomitant and adjuvant temozolomide for glioblastoma. N Engl $J$ Med 2005;352:987-96.

7 Zhang J, Stevens MFG, Bradshaw TD. Temozolomide: mechanisms of action, repair and resistance. Curr Mol Pharmacol 2012;5:102-14.

8 Auffinger B, Spencer D, Pytel P, et al. The role of glioma stem cells in chemotherapy resistance and glioblastoma multiforme recurrence. Expert Rev Neurother 2015;15:741-52.

9 Alvarado AG, Thiagarajan PS, Mulkearns-Hubert EE, et al. Glioblastoma cancer stem cells evade innate immune suppression of self-renewal through reduced TLR4 expression. Cell Stem Cell 2017;20:450-61.

10 Gimple RC, Bhargava S, Dixit D, et al. Glioblastoma stem cells: lessons from the tumor hierarchy in a lethal cancer. Genes Dev 2019;33:591-609.

$11 \mathrm{Ma}$ Q, Long W, Xing C, et al. Cancer stem cells and immunosuppressive microenvironment in glioma. Front Immunol 2018;9:2924.
12 Wakimoto H, Mohapatra G, Kanai R, et al. Maintenance of primary tumor phenotype and genotype in glioblastoma stem cells. Neuro Oncol 2012;14:132-44.

13 Cheema TA, Wakimoto H, Fecci PE, et al. Multifaceted oncolytic virus therapy for glioblastoma in an immunocompetent cancer stem cell model. Proc Natl Acad Sci U S A 2013;110:12006-11.

14 Saha D, Martuza RL, Rabkin SD. Macrophage polarization contributes to glioblastoma eradication by combination Immunovirotherapy and immune checkpoint blockade. Cancer Cell 2017;32:253-67.

15 Saha D, Martuza RL, Rabkin SD. Oncolytic herpes simplex virus immunovirotherapy in combination with immune checkpoint blockade to treat glioblastoma. Immunotherapy 2018;10:779-86.

16 Saha D, Wakimoto H, Peters CW, et al. Combinatorial effects of VEGFR kinase inhibitor axitinib and oncolytic virotherapy in mouse and human glioblastoma stem-like cell models. Clin Cancer Res 2018;24:3409-22.

17 Saha D, Wakimoto H, Rabkin SD. Oncolytic herpes simplex virus interactions with the host immune system. Curr Opin Virol 2016;21:26-34.

18 Crupi MJF, Bell JC, Singaravelu R. Concise review: targeting cancer stem cells and their supporting niche using oncolytic viruses. Stem Cells 2019;37:716-23.

19 Harrington K, Freeman DJ, Kelly B, et al. Optimizing oncolytic virotherapy in cancer treatment. Nat Rev Drug Discov 2019;18:689-706.

20 Saha D, Ahmed SS, Rabkin SD. Exploring the antitumor effect of virus in malignant glioma. Drugs Future 2015;40:739-49.

21 Bommareddy PK, Peters C, Saha D, et al. Oncolytic herpes simplex viruses as a paradigm for the treatment of cancer. Annu Rev Cancer Biol 2018;2:155-73.

22 Alonso MM, Gomez-Manzano C, Jiang H, et al. Combination of the oncolytic adenovirus ICOVIR- 5 with chemotherapy provides enhanced anti-glioma effect in vivo. Cancer Gene Ther 2007;14:756-61.

23 Gomez-Gutierrez JG, Nitz J, Sharma R, et al. Combined therapy of oncolytic adenovirus and temozolomide enhances lung cancer virotherapy in vitro and in vivo. Virology 2016;487:249-59.

24 Kanai R, Rabkin SD, Yip S, et al. Oncolytic virus-mediated manipulation of DNA damage responses: synergy with chemotherapy in killing glioblastoma stem cells. J Natl Cancer Inst 2012;104:42-55.

25 Kleijn A, van den Bossche W, Haefner ES, et al. The Sequence of Delta24-RGD and TMZ Administration in Malignant Glioma Affects the Role of $\mathrm{CD}^{+} \mathrm{T}$ Cell Anti-tumor Activity. Mol Ther Oncolytics 2017;5:11-19.

26 Garza-Morales R, Gonzalez-Ramos R, Chiba A, et al. Temozolomide enhances triple-negative breast cancer virotherapy in vitro. Cancers 2018;10:144.

27 Pisklakova A, McKenzie B, Zemp F, et al. M011L-deficient oncolytic myxoma virus induces apoptosis in brain tumor-initiating cells and enhances survival in a novel immunocompetent mouse model of glioblastoma. Neuro Oncol 2016;18:1088-98.

28 Fritzell S, Sandén E, Eberstål S, et al. Intratumoral temozolomide synergizes with immunotherapy in a T cell-dependent fashion. Cancer Immunol Immunother 2013;62:1463-74.

29 Hanihara M, Kawataki T, Oh-Oka K, et al. Synergistic antitumor effect with indoleamine 2,3-dioxygenase inhibition and temozolomide in a murine glioma model. J Neurosurg 2016;124:1594-601.

30 Ouyang $\mathrm{M}$, White $\mathrm{EE}$, Ren $\mathrm{H}$, et al. Metronomic doses of temozolomide enhance the efficacy of carbon nanotube $\mathrm{CpG}$ immunotherapy in an invasive glioma model. PLoS One 2016;11:e0148139.

31 Dai B, Qi N, Li J, et al. Temozolomide combined with PD-1 antibody therapy for mouse orthotopic glioma model. Biochem Biophys Res Commun 2018;501:871-6.

32 Marumoto T, Tashiro A, Friedmann-Morvinski D, et al. Development of a novel mouse glioma model using lentiviral vectors. Nat Med 2009;15:110-6.

33 Todo T, Martuza RL, Rabkin SD, et al. Oncolytic herpes simplex virus vector with enhanced $\mathrm{MHC}$ class I presentation and tumor cell killing Proc Natl Acad Sci U S A 2001;98:6396-401.

34 Liu T-C, Zhang T, Fukuhara H, et al. Dominant-Negative fibroblast growth factor receptor expression enhances antitumoral potency of oncolytic herpes simplex virus in neural tumors. Clin Cancer Res 2006;12:6791-9.

35 Saha D, Rabkin SD. Immunohistochemistry for tumor-infiltrating immune cells after oncolytic virotherapy. Methods Mol Biol 2020;2058:179-90.

36 Bommareddy PK, Lowe DB, Kaufman HL, et al. Multi-Parametric flow cytometry staining procedure for analyzing tumorinfiltrating immune cells following oncolytic herpes simplex virus 
immunotherapy in intracranial glioblastoma. J Biol Methods 2019;6:e112:112.

37 Rosso L, Brock CS, Gallo JM, et al. A new model for prediction of drug distribution in tumor and normal tissues: pharmacokinetics of temozolomide in glioma patients. Cancer Res 2009;69:120-7.

38 Freireich EJ, Gehan EA, Rall DP, et al. Quantitative comparison of toxicity of anticancer agents in mouse, rat, hamster, dog, monkey, and man. Cancer Chemother Rep 1966;50:219-44.

39 Spiegl-Kreinecker S, Pirker C, Filipits M, et al. O6-Methylguanine DNA methyltransferase protein expression in tumor cells predicts outcome of temozolomide therapy in glioblastoma patients. Neuro Oncol 2010;12:28-36.

40 Fan C-H, Liu W-L, Cao H, et al. O6-Methylguanine DNA methyltransferase as a promising target for the treatment of temozolomide-resistant gliomas. Cell Death Dis 2013;4:e876.

41 Murakami J, Lee Y-J, Kokeguchi S, et al. Depletion of O6methylguanine-DNA methyltransferase by 06-benzylguanine enhances 5-FU cytotoxicity in colon and oral cancer cell lines. Oncol Rep 2007;17:1461-7.

42 Platten M. How to integrate immunotherapy into standard of care in glioblastoma. Neuro Oncol 2019;21:699-700.

43 Maes W, Van Gool SW. Experimental immunotherapy for malignant glioma: lessons from two decades of research in the GL261 model. Cancer Immunol Immunother 2011;60:153-60.

44 Oh T, Fakurnejad S, Sayegh ET, et al. Immunocompetent murine models for the study of glioblastoma immunotherapy. J Trans/ Med 2014;12:107.
45 Azambuja JH, da Silveira EF, de Carvalho TR, et al. Glioma sensitive or chemoresistant to temozolomide differentially modulate macrophage protumor activities. Biochim Biophys Acta Gen Subj 2017;1861:2652-62.

46 Saha D, Martuza RL, Rabkin SD. Curing glioblastoma: oncolytic HSV-IL12 and checkpoint blockade. Oncoscience 2017;4:67-9.

47 Pessina S, Cantini G, Kapetis D, et al. The multidrug-resistance transporter ABCC3 protects NK cells from chemotherapy in a murine model of malignant glioma. Oncoimmunology 2016;5:e1108513.

48 Ogbomo $\mathrm{H}$, Cinatl $\mathrm{J}$, Mody $\mathrm{CH}$, et al. Immunotherapy in gliomas: limitations and potential of natural killer (NK) cell therapy. Trends $\mathrm{Mol}$ Med 2011;17:433-41.

49 Alvarez-Breckenridge CA, Yu J, Price R, et al. Nk cells impede glioblastoma virotherapy through NKp30 and NKp46 natural cytotoxicity receptors. Nat Med 2012;18:1827-34.

50 Karachi A, Yang C, Dastmalchi F, et al. Modulation of temozolomide dose differentially affects T-cell response to immune checkpoint inhibition. Neuro Oncol 2019;21:730-41.

51 Ning J, Wakimoto H, Peters C, et al. Rad51 Degradation: Role in Oncolytic Virus-Poly(ADP-Ribose) Polymerase Inhibitor Combination Therapy in Glioblastoma. J Natl Cancer Inst 2017;109:1-13.

52 Bai Y, Chen Y, Hong X, et al. Newcastle disease virus enhances the growth-inhibiting and proapoptotic effects of temozolomide on glioblastoma cells in vitro and in vivo. Sci Rep 2018;8:11470. 